\title{
Oregon State University Softball: Dynamic Visual Acuity Training for Improving Performance
}

\author{
Bruce Madsen MD ${ }^{1}$, Kyle Blair ${ }^{2}$ \\ 1Athletic Eye Institute \\ 2Western University of Health Sciences
}

\begin{abstract}
Sports vision training involves eye focusing and movement workouts that center on the visual tracking of objects. The purpose of sports vision training is to improve performance in various sports by improving visual responses and processing, such as by lowering reaction times. In 2015 , the Athletic Eye Institute ${ }^{L C}$ started a sports vision-training program study with the Oregon State University Softball Team in the hopes of increasing the dynamic visual skills of their players. There were two aims of this study. The first aim was to test the hypothesis that softball athletes would show improvement over time in the specific sports vision training tests. The second aim was to test the hypothesis that sports vision training would lead to improvements of offensive batting statistics from the 2015 season, when players did not have vision training, to the 2016 season, after players had begun vision training. Results showed significant improvements in the scores from the initial to final training session for eight out of ten visual training tests. However, OSU softball player offensive batting statistics showed no significant improvement from the 2015 season to the 2016 season, or compared to other teams. This study showed that performances of sports vision tests can be improved over time with training, but that these improvements may not translate into improvements in softball offensive batting statistics.
\end{abstract}

\section{Introduction}

The goal of vision training for athletes is to improve dynamic visual acuity and hand-eye coordination, with the goal of improving their overall performance in sports. Softball in particular requires a high level of dynamic visual acuity and quick reaction time in order to perform at a high level. A batter has to locate and react to a ball being thrown at speeds of averages of 59 to 64 miles per hour with only a distance of 43 feet from the pitches mound to home plate. Previous research demonstrates that batters only have $200 \mathrm{~ms}$ to make the decision to swing and another $200 \mathrm{~ms}$ to swing the bat (Flyger, Button, \& Rishiraj, 2006). If the player is able to locate the ball during the flight path and process that information quicker, the player's chances of successfully hitting the ball increase (De Lucia \& Cochran 1985; Deveau, Ozer, \& Seitz 2014; Sherwin, Muraskin, \& Sajda, 2012). Previous research suggests that athletes have a superior dynamic visual acuity to non-athletes due to a better ability to track moving objects (Uchida et al., 2013). Quicker tracking gives the player more time to decide whether to swing at the ball or not. The goal of sports vision training is improve tracking and to reduce this visual processing time so that the player can make a better decision and have more time to swing the bat.

Softball makes for an ideal sport to measure reactions and dynamic visual acuity because all players have to bat, thus there is no selection bias on picking a specific position. In many other team sports, different players play different positions, thus making it difficult to compare players of two positions, such as a quarterback to a wide receiver in football. Additionally, there are many easily quantifiable statistics in softball that can be measured. Measured statistics include runs batted in (RBI), batting average (BA), slugging percentage (SLG\%), and on-base percentage (OB\%). These four statistics reflect different aspects of offensive player ability in softball or baseball.

The softball coaching team at Oregon State University decided to work towards enhancing dynamic vision performance in 2015 so that the players might see improvements in offensive batting statistics. Research has shown improvements in baseball offensive statistics after doing sports vision training, so there was some precedent in using the training to improve dynamic visual acuity (Clark, et al., 2012). Different research has shown that vision training may not have any effect on performance in sports tasks, indicating that more research needs to be done in this area (Wood and 
Abernethy, 1997; Abernethy \& Wood, 2001). This study used a variety of different devices that other studies have not used in combination before. This is also the first study that looked at softball players specifically in relation to these training techniques, as previous sports vision-training studies have involved baseball, tennis, field hockey, and American football players. (Clark, et al., 2012; Swab \& Memmert, 2012; Clark, et al. 2015). This study aimed to determine the effectiveness of each sports vision-training test by measuring the athlete's initial and final scores. We hypothesized that athletes would see an improvement of scores in each test. This study also aimed to determine the effectiveness of the sports training test by comparing the overall batting statistics (RBI, BA, SLG\% and OB\%) from 2015 to 2016 batting statistics. We hypothesized that there would be an increase in overall batting statistics in 2016 after the sports vision training was implemented.

\section{Methods}

Participants

Participants were female softball players on the Oregon State Softball team ages to 18 to 23 who had played softball in high school. The whole team (twenty athletes) did the sports vision-training program with no exclusion criteria. Not all participants were able to complete all of the sports vision training procedures. Only eight softball players completed played in 2015 and 2016 and completed the sports vision training.

\section{Procedure}

Participants trained once or twice a week starting eight weeks prior to the beginning of the softball season and finished a week before the end of the season. Each training session lasted thirty minutes. On average, participants attended nine trainings between January 2016 and May 2016, spanning fifteen total weeks. There were no training sessions during the students' finals week and during spring break. The number of training sessions that a participant attended varied greatly, depending on their availability. Participants needed to have a minimum of two vision-training sessions in order to be included in this study. The maximum number of training sessions included in this dataset was 15 sessions. The visual training methods and tests that participants underwent were the D2 Dynavision, strobe glasses, horizontal saccades, vertical saccades, near far test, double eye speed concentration, FLIPS, PRISM, and P rotator tests.

A certified sports vision trainer collected all of the data for the vision training scores. Data from the vertical, horizontal, and oblique saccades tests were reported by the participants and then recorded. During all of the dynamic visual acuity tests, current popular music was playing so as to mimic the sound distractions the players would face while in an actual game.

Offensive performance statistics were collected from PAC-12 Conference Softball Statistics records that are available publically online (PAC-12 Softball Statistics).

\section{Vision Trainings}

\section{D2 Dynavision}

The D2 Dynavision is a 48 " by 48 " board with a total of 64 buttons that light up at different times. An LCD screen is located just above the center of the D2 board where participants focus their eyes. This is so that the participants are actively working their peripheral vision. The buttons are arranged in 5 concentric circles, similar to a target. All participants were able to reach the buttons on the outer rings without difficulty. The goal of the task is to hit the buttons when they lights up, one at a time. The participants are trained using different settings. There were three different settings in which the data was collected: the A-run, B.75, and the average reaction time settings. The D2 Dynavision collected the data on reaction times electronically.

In the A-run setting, participants had 60 seconds to hit as many buttons as possible. Buttons would light up one at a time and once a button was hit, another button would immediately light up. The benefit of this method of data collection was that reaction time could accurately be measured based on the number of buttons that were hit in 60 seconds. In the B.75 setting, each button would light up 
one at a time for a maximum of 0.75 seconds. The number of buttons that were hit (out of 80 total) determined the final score. This task lasted 60 seconds. Lastly, average reaction time was calculated from a series of different tests on the D2. These tests included hitting central, peripheral, right, and left buttons in separate tests. Current research supports the reliability of the D2 as a measure of psychomotor skills (Xi Y, Rosopa et al. 2014; Klavora, Gaskovsh, \& Forsyth, 1994; Klavora, Gaskovsh, \& Forsyth, 1995; Vesia, et al., 2008).

\section{Strobe Glasses}

Participants also practiced tossing a tennis ball to one another with strobe glasses. These glasses have LED lenses and worked by flashing black then clear when objects are in motion so that the wearer has difficulty seeing the object. This often forced the participant to predict where the ball would be based on previous visual information. The goal was that the players would be able to use this visual prediction strategy to improve their offensive batting statistics. In the lower level stages, the strobe glasses flashed rapidly while in the higher level stages the strobe glasses would flash slower. The slower the interval, the more difficult it was to catch the ball since the wearer had less time with an unimpeded image. ${ }^{16}$ The participants went up a level in difficulty after every training session. This was simply a training method and no data was collected.

\section{Reading Tests \\ Saccades}

In the horizontal saccade test, participants had to horizontally and rapidly move their eyes from one chart to another. Participants stood 3.5 feet back from two charts, which were spaced three feet apart from each other horizontally on the wall. Participants had to audibly read a letter from one chart, and then move their eyes horizontally to the other chart and audibly read the next letter in sequence, and repeat. Participants continued doing this as fast as possible for 60 seconds.

At the same time, other participants did the vertical saccade test. In this test, participants did the same procedure but the charts were spaced three feet apart from each other vertically on the wall. Multiple people did the saccade test at the same time in order to add distraction. The score was measured by how many total letters were read in the 60 second timeframe.

\section{Near Far Test}

The Near Far Test was similar to the saccade test. Participants went back and forth between a small card 8 inches from their face and a paper 4 feet away. The participant had to read a letter on the card then read the next letter in the sequence on the paper on the wall, and repeat. The goal was to go back and forth reading letters from the card to the sheet on the wall as fast as possible for 60 seconds. The score was measured by how many total letters were read in 60 seconds.

\section{P-rotator}

The P-rotator is an electronically controlled rotating disk. On the disk there were letters attached by Velcro scattered throughout. While the disk is rotating clockwise at a constant speed, participants have to find each letter in a specific order. Scores were measured by how many letters the participant found in 60 seconds.

\section{Double Eye Speed Concentration (DESC)}

In the double eye speed concentration tests, there were two boards with numbers scattered throughout. The goal was to find a number on the first board and then find the same number on the second board. Participants went in numerical order from one to 40 and tried to get as far as they could in 60 seconds. The DESC test was scored based on how many numbers the athlete reported they found in 60 seconds.

\section{PRISM and FLIPS Glasses}

The PRISM and FLIPS glasses had two pairs of lenses, one set on top and one set on bottom. The glasses had a handle in which the participants could flip between the lenses quickly. In the PRISM glasses, the top lenses were base in and the bottom lenses were base out, both at a power of 2.00 
prism diopters. This forced the wearer to alternate moving the eyes outward and inward to focus. In the FLIPS glasses test, the top and bottom lenses alternated between plus 1.50 and minus 1.50 diopters, forcing the wearer to alternate focusing far away then up close. In both tests, participants read a chart with seven columns of three letters each and seven rows of 24 letters as fast as possible. After the participant read a column of three letters out loud, she then had to flip to the other set of lenses and read the next column of three letters. The goal was to read as many columns as possible in 60 seconds. The PRISM and FLIPs tests were scored based on how many letters the athlete reported they read in 60 seconds.

\section{Statistical Methods}

Visual test scores were collected at each training session. Paired t-tests were used to assess significant differences between each player's first recorded test score and last recorded test score (Table 1). Assumptions were checked both visually and using the Anderson-Darling test for normality. The mean difference from initial to final score and 95\% Confidence Intervals for the mean difference were calculated and reported in Table 1 for each visual test.

Offensive batting statistics (batting average, runs brought in, slugging percentage, and on-base percentage) were collected for all PAC-12 division softball players that played in both the 2015 and 2016 softball seasons. Changes in performance statistics from 2015 to 2016 for OSU players were assessed using paired t-tests. Assumptions were checked both visually and using the AndersonDarling test for normality. The mean difference from 2015 performance to 2016 performance and 95\% Confidence Intervals for the mean difference were calculated and reported in Table 2 for OSU players. Additionally, changes in offensive batting statistics for OSU were compared to changes in offensive batting statistics for all other team players using two sample t-tests. Again, assumptions were checked visually, using the Anderson-Darling test for normality and Levene's Test for checking for equal variance.

\section{Results}

Table 1 summarizes changes in visual-training test scores for all OSU softball athletes. When comparing the first training session to the final training session, improvements were seen in the D2 Dynavision tests (A-run, B.75, average reaction time), in the PRISM and FLIPS glasses tests, and in the horizontal saccades, vertical saccade, and near far reading tests (Table 1). Tests that did not show statistically significant improvement included the double eye speed concentration (DESC) test and the P-rotator reading test.

Table 1. Changes in visual training test scores from initial to final training

\begin{tabular}{|c|c|c|c|c|}
\hline Visual Training Test & $\begin{array}{c}\text { Mean Initial } \\
\text { Score (SD) } \\
\end{array}$ & $\begin{array}{r}\text { Mean Final } \\
\text { Score (SD) } \\
\end{array}$ & $\begin{array}{l}\text { Difference in Mean Score } \\
\text { (95\% Confidence Interval) }\end{array}$ & P-value \\
\hline \multicolumn{5}{|l|}{ D2 Dynavision } \\
\hline A-run $(\mathrm{N}=13)$ & $75.9(9.1)$ & $101.3(6.7)$ & $+25.5 \quad(18.8,32.1)$ & $\mathrm{p}<0.001^{*}$ \\
\hline B.75 $(\mathrm{N}=16)$ & $67.8(11.1)$ & $88.4(9.6)$ & $+20.7 \quad(12.5,28.9)$ & $\mathrm{p}<0.001^{*}$ \\
\hline Average Reaction Time $(\mathrm{N}=20)$ & $0.80(0.09)$ & $0.35 \quad(0.03)$ & $-0.45 \quad(-0.49,-0.40)$ & $\mathrm{p}<0.001 *$ \\
\hline \multicolumn{5}{|l|}{ Reading Tests } \\
\hline Horizontal saccade $(\mathrm{N}=14)$ & $32.4(5.0)$ & $48.9(7.9)$ & $+16.4(13.5,19.4)$ & $\mathrm{p}<0.001^{*}$ \\
\hline Vertical saccade $(\mathrm{N}=14)$ & $32.3(5.1)$ & $43.9(8.1)$ & $+11.6(6.7,16.6)$ & $\mathrm{p}<0.001^{*}$ \\
\hline Near Far Test $(\mathrm{N}=12)$ & $34.8(6.2)$ & $52.9(7.9)$ & $+18.1(14.0,22.2)$ & $\mathrm{p}<0.001 *$ \\
\hline P-rotator $(\mathrm{N}=12)$ & $7.1 \quad(2.8)$ & $9.1 \quad(2.4)$ & $+2.0 \quad(-0.2,4.2)$ & $\mathrm{p}=0.075$ \\
\hline \multicolumn{5}{|l|}{$\begin{array}{l}\text { Double Eye Speed } \\
\text { Concentration (DESC) }\end{array}$} \\
\hline DESC $(\mathrm{N}=16)$ & $17.13(3.4)$ & $17.56(2.6)$ & $+0.44 \quad(-1.7,2.6)$ & $\mathrm{p}=0.67$ \\
\hline \multicolumn{5}{|l|}{ PRISM \& FLIPS Glasses } \\
\hline PRISM (N=11) & $37.5(12.1)$ & $66.5(15.9)$ & $+29.0 \quad(21.7,36.3)$ & $\mathrm{p}<0.001^{*}$ \\
\hline FLIPS $(\mathrm{N}=12)$ & $39.0(9.2)$ & $63.2(17.9)$ & $+24.2 \quad(14.2,34.2)$ & $\mathrm{p}<0.001^{*}$ \\
\hline
\end{tabular}


Table 1 compares visual training test scores in softball athletes. Paired t-tests were used to compare each athlete's first recorded test score to their last recorded test score. A $*$ indicates significant differences in test scores at an alpha level of 0.05

Table 2 compares OSU softball performance statistics from 2015 to performance in 2016. Players were only included in this analysis if they played in both the 2015 and 2016 season. The OSU players showed improvement in offensive statistics from the 2015 to the 2016 season, but these improvements were not statistically significant.

Table 2. Changes in OSU offensive performance statistics from 2015 season to 2016 season

\begin{tabular}{lccccc}
\hline & $\begin{array}{c}\text { Mean } \\
\text { Performance } \\
\mathbf{2 0 1 5}\end{array}$ & $\begin{array}{c}\text { Mean } \\
\text { Performance } \\
\mathbf{2 0 1 6}\end{array}$ & $\begin{array}{c}\text { Difference in Mean } \\
\text { Performance }\end{array}$ & \\
& (95\% Confidence Interval) & P-value \\
\hline Batting Average (N=8) & $0.22(0.14)$ & $0.28(0.08)$ & $+0.064(-0.06,0.19)$ & $\mathrm{p}=0.274$ \\
RBI (N=8) & $18.6(14.6)$ & $20.5(16.4)$ & $+1.88(-15.70,19.45)$ & $\mathrm{p}=0.808$ \\
SLG\% (N=8) & $0.33(0.22)$ & $0.42(0.13)$ & $+0.09(-0.14,0.31)$ & $\mathrm{p}=0.397$ \\
OB\% (N=8) & $0.36(0.09)$ & $0.38(0.06)$ & $+0.01(-0.09,0.12)$ & $\mathrm{p}=0.762$ \\
\hline
\end{tabular}

Table 2 compares OSU softball player performance statistics in 2015 to their performance in 2016. Performance statistics were compared using paired ttests. No significant differences were found at an alpha level of 0.05

Additional data on performance statistics was gathered for all other PAC-12 conference softball players for the 2015 and 2016 seasons. Again, players who were in both the 2015 and 2016 seasons were included. Two sample t-tests were used to compare changes in OSU player performance to other team players. No significant differences were observed at an alpha level of 0.05

\section{Discussion}

This study did not show attributable improvements in offensive performance statistics from 2015 to 2016. This finding falls in line with similar research showing that visual training does not improve visual or motor performance (Wood and Abernethy, 1997; Abernethy 7 Wood, 2001). However, the improvement of the team in all of the offensive statistics is an encouraging sign, especially since the rest of the PAC 12 showed a decrease in these categories. Since other studies have shown statistically significant improvement in these percentages (Clark et al. 2010; Clark et al. 2012), the data does not necessarily indicate that the sports vision training had no effect at all. There are many other factors that could play a role in the improvement in these percentages in the Oregon State Softball team. Continued data collection with more participants will be important in supporting that the vision training has a positive impact on batting outcomes.

Athlete improvement in the performance over time in the tests themselves agrees with previous research that shows that athletes can be trained to improve dynamic visual acuity (Zwierko, et al. 2015). This is important, since without showing improvement, a case could not be made that the vision training caused improvement in sports performance. Our recommendation is that D2 Dynavision, horizontal saccades, vertical saccades, near far, PRISM, and FLIPS tests be used in future studies, as they showed improvement in scores after multiple trials. Not all training methods showed reliable athlete improvement. The DESC test will be dropped from the visual training curriculum due to lack of athlete improvement. However, the P-rotator test will be continued, despite lack of significant improvement of scores. With this information, we hope to help direct future vision training programs to improve sports vision performance.

\section{Limitations}

The biggest limitation in this study is the limited number of participants from 2015 to 2016. Only eight players played in both years, reducing the power of the study significantly. Data will continue to be collected for the OSU softball team each year to achieve a large enough sample size and to have adequate power in the study. Additionally, this study took place after only one season of visual 
training occurred, so it is possible that offensive performance statistics could show improvement in future seasons after additional training has occurred.

Another limitation of this study was that the comparison of offensive performance statistics between the OSU softball players and the rest of the PAC-12 softball players was not ideal. There are many confounding factors that could have accounted for the overall changes in offensive statistics of the OSU softball players compared to the rest of the PAC- 12 teams. Some of these confounders are including but not limited to different schedules, training programs, coaches, home fields, roster changes, etc. An ideal study would randomly select players to undergo sports vision training and compare them to players that did not undergo sports vision training.

The inconsistency of the visual training was another limitation of this study. The vision training sessions had to be worked around the schedule of college students, which was often quite busy. The timing of these sessions was not consistent every week. It would be valuable to improve the consistency of training of the athletes by ensuring athletes participated in an appropriate number of training sessions and went to training at approximately the same times.

\section{Conclusion}

Some sports vision training research has shown promise towards improving performance in sports tasks, but other research has shown that sports vision training may not have any effect on performance in sports tasks. This study does not support the assertion that sports vision training improves offensive performance statistics in softball players. However, athletes did show significant improvement in visual test performance in eight out of the ten tests. The small sample size is a major limitation of these results, and future studies will be needed to increase the study power and further explore the relationship between vision training and performance in softball athletes at Oregon State University.

\section{Acknowledgments}

The authors acknowledge and thank Samaritan Athletic Center for the use of the facilities. The authors would also like to thank Graham Erickson for his input and contribution to this paper.

\section{Funding}

No sources of funding were used to create this paper

\section{Conflict of interest}

Bruce Madsen MD and Kyle Blair declare that they have no conflicts of interests relevant to the contents of this paper.

Designers of the program

Bruce Madsen MD, Ophthalmologist

Donald Pennington MD, Orthopedic Surgeon

http://www.athleticeyeinstitute.com

541-768-7980 | info@athleticeyeinstitute.com 


\section{References}

1. Flyger N1, Button C, Rishiraj N. (2006). The science of softball: implications for performance and injury prevention. Sports Med, 36(9):797-816.

2. De Lucia PR, Cochran EL. (1985). Perceptual information for batting can be extracted throughout a ball's trajectory. Percept Mot Skills, 61(1):143-50.

3. Deveau J, Ozer DJ, Seitz AR. (2014). Improved vision and on-field performance in baseball through perceptual learning. Cur Biol, 24(4):146-47

4. Sherwin, J., Muraskin, J., \& Sajda, P. (2012) You Can't Think and Hit at the Same Time: Neural Correlates of Baseball Pitch Classification. Front Neurosci, 6:177.

5. Uchida Y, et al. (2013) Dynamic Visual Acuity in Baseball Players Is Due to Superior Tracking Abilities. Med Sci Sports Exerc, 45(2):319-325.

6. Clark JF, Ellis JK, Bench J, Khoury J, Graman P. (2012) High-Performance Vision Training Improves Batting Statistics for University of Cincinnati Baseball Players. PLOS ONE, 7(1):1-6.

7. Wood JM and Abernethy, B. (1997). An assessment of the efficacy of sports vision training programs. Optom Vis Sci, 74:646-659.

8. Abernethy B, and Wood JM. (2001). Do generalized visual training programs for sport really work? An experimental investigation. J Sports Sci, 19:203-22.

9. Swab S, Memmert D. (2012) The impact of a sports vision training program in youth field hockey players. J Sports Sci Med, 11:624-31.

10. Clark, JF et al. (2015) An Exploratory Study of the Potential Effects of Vision Training on Concussion Incidence in Football. Optom Vis Perform, 3(2):116-25.

11. PAC 12. PAC-12 Conference Softball Statistics: PAC-12 Conference Softball. Available at: http://pac12.com/content/softball-statistics [accessed 08.23.2016].

12. Xi Y, Rosopa PJ, Mossey M, Crisler MC, Drouin N, Kopera K, \& Brooks JO. (2014). The Reliability of a VISION COACH Task as a Measure of Psychomotor Skills. Occup Ther Health Care, 28(4):1-11

13. Klavora P, Gaskovsh P, \& Forsyth RD (1994). Test-retest reliability of three Dynavision tasks. Percept Mot Skills, 80(2):607-10.

14. Klavora P, Gaskovski P, \& Forsyth RD. (1995). Test-retest reliability of the Dynavision apparatus. Percept Mot Skills, 79(1):448-50.

15. Vesia M, Esposito J, Prime SL, Klavora P. (2008) Correlations of selected psychomotor and visuomotor tests with initial Dynavision performance. Percept Mot Skills. 107(1):14-20.

16. Kirscher DW. (1993) Sports vision training procedures. Optom Clin, 3(1):171-82.

17. Zwierko T, et al. (2015). The Effects of Sports Vision Training on Binocular Vision Function in Female University Athletes. J Hum Kinet, 49:287-96. 